\title{
Updated checklist of the Cantharidae, Lampyridae, and Lycidae of the Grand Duchy of Luxembourg (Coleoptera: Elateroidea)
}

\author{
FABRIZIO FANTI ${ }^{1} \&$ FRANCESCO VITALI $^{2}$ \\ ${ }^{1}$ Via del Tamburino 69, I-53040 Piazze (Siena), Italy. \\ "Eantifab@alice.it; @ https://orcid.org/0000-0003-2002-108X \\ ${ }^{2}$ National Museum of Natural History, rue Münster 24, L-2160 Luxembourg, Luxembourg. \\ Corresponding author. $\risingdotseq$ fvitali@mnhn.lu; 이ttps://orcid.org/0000-0003-3052-2910
}

\begin{abstract}
Here we present a checklist of the families Cantharidae, Lampyridae, and Lycidae of Luxembourg. The earlier references are summarized and new information on the some species is reported. Furthermore, Cantharis (s. str.) paludosa Fallén, 1807, Cantharis (s. str.) terminata Faldermann, 1835, Erotides cosnardi (Chevrolat, 1831), and Lopheros rubens (Gyllenhal, 1817) are cited for the first time for Luxembourg based on specimens preserved in the National Museum of Natural History or on a photo reported on the iNaturalist platform.
\end{abstract}

Key words: scientific heritage, museum collections, soldier beetles, fireflies, net-winged beetles

\section{Introduction}

Despite the basic importance of national checklists, it has been well known since Mousset's (1977) times that Luxembourg has not a complete faunistic knowledge of its territory yet. Only in recent years, a more careful management of economic and human resources has allowed the realization of these fundamental studies. Though atlases or checklists on Heteroptera (Reichling 2001), Orthoptera (Proess 2004), Odonata (Proess 2006), Auchenorrhyncha (Niedringhaus et al. 2010), Hymenoptera (Chevin et al. 2011; Wegnez et al. 2021) and many families of Lepidoptera (Meyer \& Pelles 1981, 1989; Hellers 2016, 2017, 2018, 2019, 2020) were realised, Coleoptera were rarely studied (Mousset 1969; Braunert 1996, 2009; Braunert \& Gerend 1997; Gerend 2003, 2006; Vitali 2012, 2018).

The cantharoid families, and in particular the soldier beetles (Cantharidae), have been little taken into account throughout Europe and only recently, some countries have seen the drafting of catalogues or checklists: Russia (Kazantsev 2004), Spain (Diéguez-Fernández 2011, 2012, 2014), United Kingdom (Alexander 2012), Italy (Fanti 2014) and France (Constantin 2014).

However, only sparse short notes (Magis 1956, 1970, 1971, 1972, 2005; Meyer 1977; Mousset 1981a, 1981b; Gerend 2000; Bretzendorfer 2002; Gerend et al. 2007; Köhler 2009, 2011, 2013) can be found for Luxembourg. The checklist of the Palaearctic fauna of Cantharidae (Kazantsev \& Brancucci 2007) reported for this country only 27 species, and that list is incomplete as we could see after a check of the local literature.

Lampyridae in Luxembourg have been similarly poorly studied. The recent Palaearctic catalogue (Geisthardt \& Satö 2007) mentions only two species, overlooking past records (Magis 1976, 1977; Gerend 2000) of Lamprohiza splendidula (Linnaeus, 1767).

Finally, Bocakova \& Bocak (2007) based on the primary source of Bocak \& Bocakova (1987) mentioned no species of Lycidae for Luxembourg in the Palaearctic catalogue, though at least two species were known (Mousset 1981b; Magis 1976, 1977).

The aim of this publication is to assess the situation of these families in the Grand Duchy of Luxembourg in order to attract the interest of local and international collaborators. The final goal is the realisation of a colour atlas focused on these families, including distribution maps, phenology of the local species and analyses of the faunistic changes. 


\section{Materials and Methods}

The present study is based on the literature records concerning Luxembourg (including hunting notes and published meeting relations of the Luxembourg Naturalist Society), the beetle collection deposited in the National Museum of Natural History, Luxembourg (MNHNL), the authors' collections and the photographs obtained from the citizen science platform iNaturalist (https://www.inaturalist.org). All species are reported in alphabetical-systematic order, with the binomial in use accompanied by author and year of description, as well as the full reference works for Luxembourg.

Photographs of the specimens were taken with a CMOS Camera mounted on a Keyence VHX 6000 digital microscope equipped with a VHX-S660E free-angle observation system, a VH-ZST 20-2000x double zoom objective, 2D/3D image stitching system and stacking system taking to 200 images at 2 million pixels of resolution, owned by the MNHNL. All photographs were enhanced with the Ulead PhotoImpact SE software.

\section{Results}

\section{FAMILY Cantharidae Imhoff, 1856}

Ancistronycha abdominalis (Fabricius, 1798)

Magis 2005.

Ancistronycha violacea (Paykull, 1798)

Ferrant 1917; Magis 1956, 1968, 1971; Kazantsev \& Brancucci 2007; Kazantsev 2012.

Cantharis (s. str.) cryptica Ashe, 1947

Magis 1968; Gerend et al. 2007; Kazantsev \& Brancucci 2007; Köhler 2009, 2011, 2013; Švihla \& Dvořák 2009

[Kazantsev \& Brancucci 2007]; Kazantsev 2012.

Cantharis (s. str.) decipiens Baudi di Selve, 1872

Magis 1968; Gerend et al. 2007; Kazantsev \& Brancucci 2007; Köhler 2009, 2011, 2013; Kazantsev 2012.

Cantharis (s. str.) figurata Mannerheim, 1843

Magis 1968; Kazantsev \& Brancucci 2007; Kazantsev 2012.

Cantharis (s. str.) flavilabris Fallén, 1807

Köhler 2009, 2011 (sub Cantharis fulvicollis).

Cantharis (s. str.) fusca Linnaeus, 1758

Olm 1892; Ferrant 1917; Magis 1956, 1968, 1970, 1972; Mousset 1981a; Gerend 2000; Gerend et al. 2007; Kazantsev \& Brancucci 2007; Köhler 2011, 2013; Kazantsev 2012.

Cantharis (s. str.) livida Linnaeus, 1758

Magis 1956, 1968, 1971; Gerend et al. 2007; Kazantsev \& Brancucci 2007; Silkin 2008; Kazantsev 2012; Köhler 2013.

Cantharis (s. str.) nigra (De Geer, 1774)

Köhler 2013 (sub Cantharis thoracica).

Note. C. nigra is often and easily confused with Cantharis flavilabris. The presence and distribution in Luxembourg need to be further studied.

Cantharis (s. str.) nigricans (O. F. Müller, 1776)

Magis 1956, 1968, 1971; Kazantsev \& Brancucci 2007; Köhler 2009, 2011; Kazantsev 2012. 
Cantharis (s. str.) obscura Linnaeus, 1758

Magis 1956, 1968; Gerend et al. 2007; Kazantsev \& Brancucci 2007; Köhler 2009; Kazantsev 2012.

Cantharis (s. str.) pallida Goeze, 1777

Mousset 1981a; Köhler 2009, 2011, 2013.

Cantharis (s. str.) paludosa Fallén, 1807 (Fig. 1)

Material. Wincrange, Hoffelt, 8.VI.2000, A. Mousset leg. [MNHNL].

Note. C. paludosa is a hygrophilous species, not rare in humid environments.

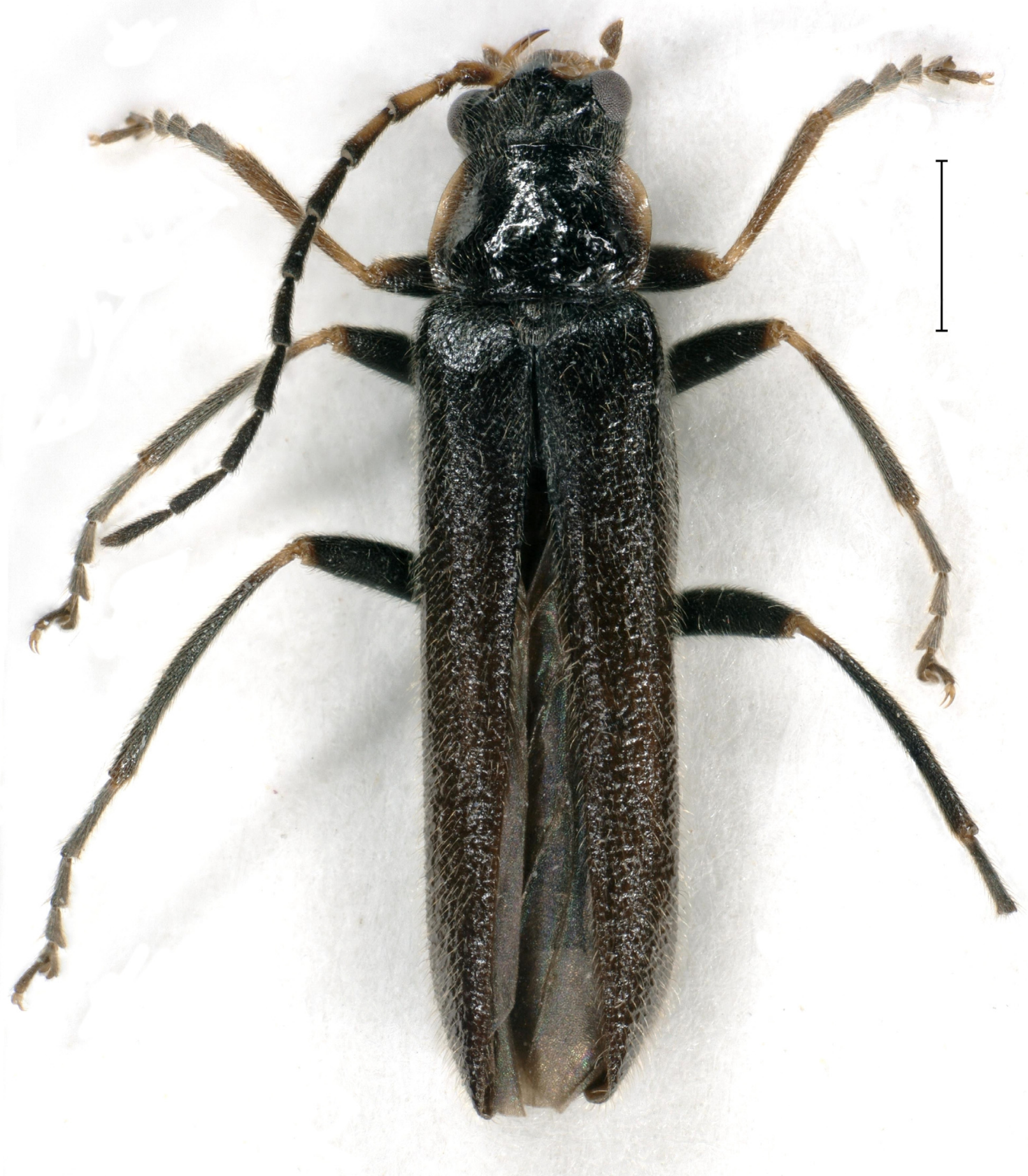

FIGURE 1. Cantharis paludosa Fallén, 1807. Specimen preserved at the National Museum of Natural History of Luxembourg, scale bar $=1.0 \mathrm{~mm}$. 
Cantharis (s. str.) paradoxa Hicker, 1960

Gerend et al. 2007; Köhler 2009; Zagatti \& Mériguet 2014 (Benelux).

Cantharis (s. str.) pellucida Fabricius, 1792

Magis 1956, 1968, 1971; Mousset 1981a; Gerend et al. 2007; Kazantsev \& Brancucci 2007; Köhler 2009, 2011, 2013.

Cantharis (s. str.) pulicaria Fabricius, 1781

Magis 1956; Kazantsev \& Brancucci 2007; Silkin 2008; Kazantsev 2012.

Note. C. pulicaria is a rather rare species throughout the distributional range. We do not know any collection specimen from Luxembourg, so the actual distribution in the Grand Duchy must be confirmed.

Cantharis (s. str.) rufa Linnaeus, 1758

Olm 1892; Magis 1956, 1968; Mousset 1981a; Gerend 2000; Kazantsev \& Brancucci 2007; Silkin 2008; Kazantsev 2012; Köhler 2011, 2013.

Note. Reported as Cantharis (s. str.) darwiniana (Sharp, 1867): Kazantsev \& Brancucci 2007; Kazantsev 2012.

Cantharis (s. str.) rustica Fállen, 1807

Magis 1956, 1968, 1971, 1972; Kazantsev \& Brancucci 2007; Silkin 2008; Kazantsev 2012; Köhler 2013.

Cantharis (s. str.) terminata Faldermann, 1835 (Fig. 2)

Material. Luxembourg Hollerich, 10.VI.1975, C. Hahn leg., 1 spec. [MNHNL]; Strassen, 13.IX.1998, A. Mousset leg., 1 spec. [MNHNL]; Strassen, Ruderlech, on Tilea, 24.V.2009, F. Vitali leg., 1 spec. [coll. F. Vitali]; Luxembourg Bambësch, 17.V.2014, F. Vitali, 1 spec. [coll. F. Vitali].

Note. C. terminata is a rare montane species, similar to Cantharis quadripunctata (O. F. Müller, 1776) and C. montana Stierlin, 1898 but basically with a black elytral margin. The species is widespread in Europe and the Middle East, and common in Belgian Luxembourg (Everts 1922; Horion 1953).

Cantharis (Cyrtomoptila) lateralis Linnaeus, 1758

Magis 1968, 1971; Kazantsev \& Brancucci 2007; Silkin 2008; Kazantsev 2012; Köhler 2013.

Cantharis (Cyrtomoptila) pagana Rosenhauer, 1847

Magis 1968, 1971; Švihla 2006; Gerend et al. 2007; Kazantsev \& Brancucci 2007; Köhler 2009; Kazantsev 2012; Fanti 2014.

Cratosilis denticollis (Schummel, 1844)

Magis 1968, 1971; Kazantsev \& Brancucci 2007; Köhler 2011; Kazantsev 2012.

Metacantharis clypeata (Illiger, 1798)

Köhler 2011.

Podistra (Absidia) rufotestacea (Letzner, 1845)

Gerend et al. 2007 (sub Absidia rufotestacea); Köhler 2009, 2011 (sub Absidia rufotestacea), 2013 (sub Absidia rufotestacea).

Rhagonycha (s. str.) elongata (Fállen, 1807)

Magis 1956; Kazantsev \& Brancucci 2007; Kazantsev 2012.

Note. This species is often confused with Rhagonycha gallica Pic, 1923 and Rhagonycha atra (Linnaeus, 1767). We do not know collection specimens from Luxembourg; therefore, its actual presence must be verified. 


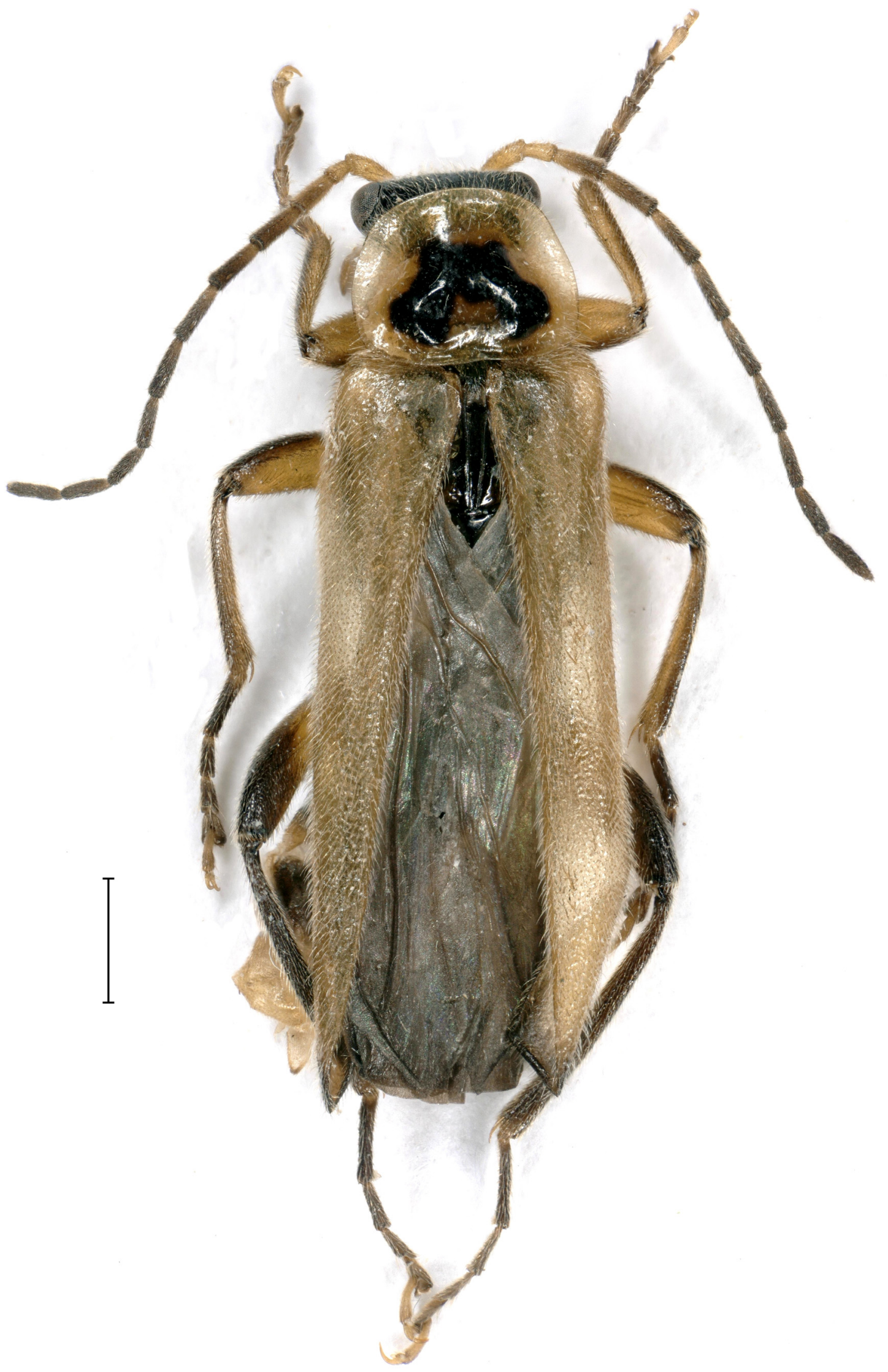

FIGURE 2. Cantharis terminata Faldermann, 1835. Specimen preserved at the National Museum of Natural History of Luxembourg, scale bar $=1.0 \mathrm{~mm}$. 
Rhagonycha (s. str.) fulva (Scopoli, 1763)

Magis 1956, 1968, 1971; Meyer 1977; Mousset 1981a (sub Cantharis fulva); Gerend et al. 2007; Kazantsev \& Brancucci 2007; Silkin 2008; Köhler 2009, 2011, 2013; Kazantsev 2012.

Rhagonycha (s. str.) gallica Pic, 1923

Gerend et al. 2007; Kazantsev \& Brancucci 2007; Köhler 2009, 2011; Kazantsev 2012.

Rhagonycha (s. str.) lignosa (O. F. Müller, 1764)

Magis 1956, 1968, 1971; Gerend et al. 2007; Kazantsev \& Brancucci 2007; Köhler 2009, 2011, 2013; Kazantsev 2012.

Rhagonycha (s. str.) lutea (O. F. Müller, 1764)

Magis 1956, 1971; Gerend et al. 2007; Kazantsev \& Brancuccci 2007; Silkin 2008; Köhler 2011, 2013.

Rhagonycha (s. str.) nigriventris Motschulsky, 1860

Magis 1956 (sub limbata), 1968 (sub limbata), 1971 (sub limbata); Mousset 1981a (sub limbata); Gerend 2000 (sub limbata); Gerend et al. 2007 (sub limbata); Kazantsev \& Brancucci 2007; Köhler 2011 (sub limbata), 2013 (sub limbata); Kazantsev 2012.

Rhagonycha (s. str.) testacea (Linnaeus, 1758)

Magis 1968, 1971; Kazantsev \& Brancucci 2007; Köhler 2009, 2011, 2013; Kazantsev 2012.

Rhagonycha (s. str.) translucida (Krynicki, 1832)

Köhler 2011.

Podabrus alpinus (Paykull, 1798)

Magis 1956, 1968, 1970; Gerend et al. 2007; Kazantsev \& Brancucci 2007; Köhler 2009; Kazantsev 2012.

Malthinus (s. str.) balteatus Suffrian, 1851

Köhler 2009.

Malthinus (s. str.) bilineatus Kiesenwetter, 1852

Kazantsev \& Brancucci 2007; Kazantsev 2012; Chittaro et al. 2021.

Malthinus (s. str.) facialis C. G. Thomson, 1864

Gerend et al. 2007; Köhler 2009.

Malthinus (s. str.) fasciatus (A. G. Olivier, 1790)

Gerend et al. 2007.

Malthinus (s. str.) flaveolus (Herbst, 1786)

Gerend et al. 2007 (sub Malthinus punctatus); Köhler 2009, 2011 (sub Malthinus punctatus), 2013 (sub Malthinus punctatus).

Malthinus (s. str.) frontalis (Marsham, 1802)

Köhler 2009, 2011.

Malthinus (s. str.) glabellus Kiesenwetter, 1852

Köhler 2011, 2013.

Malthinus (s. str.) seriepunctatus Kiesenwetter, 1852

Köhler 2011, 2013.

$38 \cdot$ Zootaxa 5047 (1) (C) 2021 Magnolia Press

FANTI \& VITALI 
Malthodes (s. str.) alpicola Kiesenwetter, 1852

Bretzendorfer 2002.

Malthodes (s. str.) fuscus (Waltl, 1838)

Gerend et al. 2007.

Malthodes (s. str.) guttifer Kiesenwetter, 1852

Gerend et al. 2007.

Malthodes (s. str.) marginatus (Latreille, 1806)

Gerend et al. 2007; Köhler 2009, 2011, 2013.

Malthodes (s. str.) maurus (Laporte de Castelnau, 1840)

Magis 1971.

Malthodes (s. str.) minimus (Linnaeus, 1758)

Magis 1956, 1971; Kazantsev \& Brancucci 2007; Köhler 2011, 2013; Kazantsev 2012.

Malthodes (s. str.) misellus Kiesenwetter, 1852

Magis 1956 (sub Malthodes maurus var. misellus); Kazantsev \& Brancucci 2007.

Malthodes (s. str.) mysticus mysticus Kiesenwetter, 1852

Gerend et al. 2007.

Malthodes (s. str.) pumilus (Brébisson, 1835)

Köhler 2013.

Malthodes (s. str.) spathifer Kiesenwetter, 1852

Magis 1968, 1971; Kazantsev \& Brancucci 2007; Köhler 2011, 2013.

\section{Malthodes sp.}

Gerend et al. 2007; Köhler 2009, 2011, 2013.

Note. Female specimens cannot be reliably identified as the diagnostic characters are limited to the shape of the terminal male tergites and sternites.

\section{FAMILY Lampyridae Rafinesque, 1815}

Lamprohiza splendidula (Linnaeus, 1767)

Magis 1976, 1977; Gerend 2000; Gerend et al. 2007; Köhler 2009, 2013.

Lampyris noctiluca (Linnaeus, 1758)

Magis 1956, 1976, 1977; Geisthardt \& Satö 2007; Geisthardt 2012 [2007]; Köhler 2013.

Phosphaenus hemipterus (Goeze, 1777)

Geisthardt \& Satö 2007; Majka \& MacIvor 2009; Köhler 2011; Geisthardt 2012 [2007].

FAMILY Lycidae Laporte, 1836

Lygistopterus sanguineus (Linnaeus, 1758)

Fanti \& Vitali 2013. 
Dictyoptera aurora (Herbst, 1784)

Mousset 1981b; Köhler 2009, 2013.

Erotides cosnardi (Chevrolat, 1831) (Fig. 3)

Material. Ahn, VII.[ 1900], V. Ferrant leg., 2 specimens [MNHNL].

Lopheros rubens (Gyllenhal, 1817)

Material. Boevange-sur-Attert, Rue Knäppchen, 9.V.2020, haeau979_5c2, 1 specimen, (https://www.inaturalist.org/ observations/45360773).

Platycis (s. str.) minuta (Fabricius, 1787)

Magis 1976, 1977; Gerend et al. 2007; Köhler 2011, 2013.

Pyropterus nigroruber (De Geer, 1774)

Köhler 2009, 2011.

\section{Discussion}

The first observations of the soldier beetles of the Grand Duchy of Luxembourg were reported by Olm (1892) and Ferrant (1917), but the local fauna of the family was thoroughly studied only in the second half of the $20^{\text {th }}$ century (Magis 1956, 1968, 1970, 1971), sometimes only marginally (Mousset 1981a; Gerend et al. 2007; Köhler 2013). Currently, 51 species of Cantharidae are known for the Grand Duchy of Luxembourg. Indeed, 22 species overlooked (Magis 1971, 2005; Mousset 1981a; Bretzendorfer 2002) or published later (Gerend et al. 2007; Köhler 2009, 2011, 2013), as well as others reported here, must be added to the 27 species reported by the most recent list of Palaearctic fauna (Kazantsev \& Brancucci 2007).

Cantharis pulicaria and Rhagonycha elongata were mentioned for Luxembourg (Magis 1956) but being difficult to recognize or with many problematic data along the range, their records must be verified and their present distribution studied in detail. Furthermore, Cantharis nigra, recently added to the Luxembourg fauna (Köhler 2013), must be verified with specimens identified by the careful study of male genitalia to avoid the confusion with $C$. flavilabris. In addition, we have not seen any specimen of some species (Malthinus balteatus, Malthinus bilineatus, Malthodes alpicola, Malthodes misellus, Malthodes mysticus, Malthodes pumilus), which putatively occur in Luxembourg and might be are preserved in private collections. In this case, however, authors' determination and their presence in Luxembourg are not in question, being also well distributed in the neighboring territories.

Many species have been cited for Luxembourg after the 2000s, indicating that the local fauna of Cantharidae is still unsatisfactory studied. In fact, other species, e.g., Cantharis quadripunctata, Cantharis montana, Metacantharis discoidea, Rhagonycha nigriceps, Malthinus biguttatus, Malthodes dispar, could be found and listed in the future.

Concerning Lampyridae, the knowledge is complete, with the recent mention of Phosphaenus hemipterus (Geisthardt \& Satö 2007; Majka \& MacIvor 2009; Köhler 2011; Geisthardt 2012). Phosphaenus hemipterus was probably overlooked to the small body size and biology. The species is found under stones and on walls in wooded and rural areas. In additions, the males communicate by pheromones and not by the bioluminescence.

Finally, no Lycidae species was reported for Luxembourg in the Palaearctic catalogue (Bocakova \& Bocak 2007), although the species known from western Europe are widely distributed (Bocak \& Bocakova 1987). Only in recent years or with the present work, some species have been reported from Luxembourg and the knowledge is almost complete. It should be noted that the record of Erotides cosnardi from Luxembourg is based on two specimens collected around 1900 but that the species has never been mentioned in the literature. This underlines the importance of the collections, their preservation and their study with modern criteria.

\section{Acknowledgements}

We are grateful to the anonymous reviewers. The present publication is financed by the Museum of Natural History, Luxembourg through a triennial commitment (201902537-scie, 202002892-scie, 202103198-scie). 


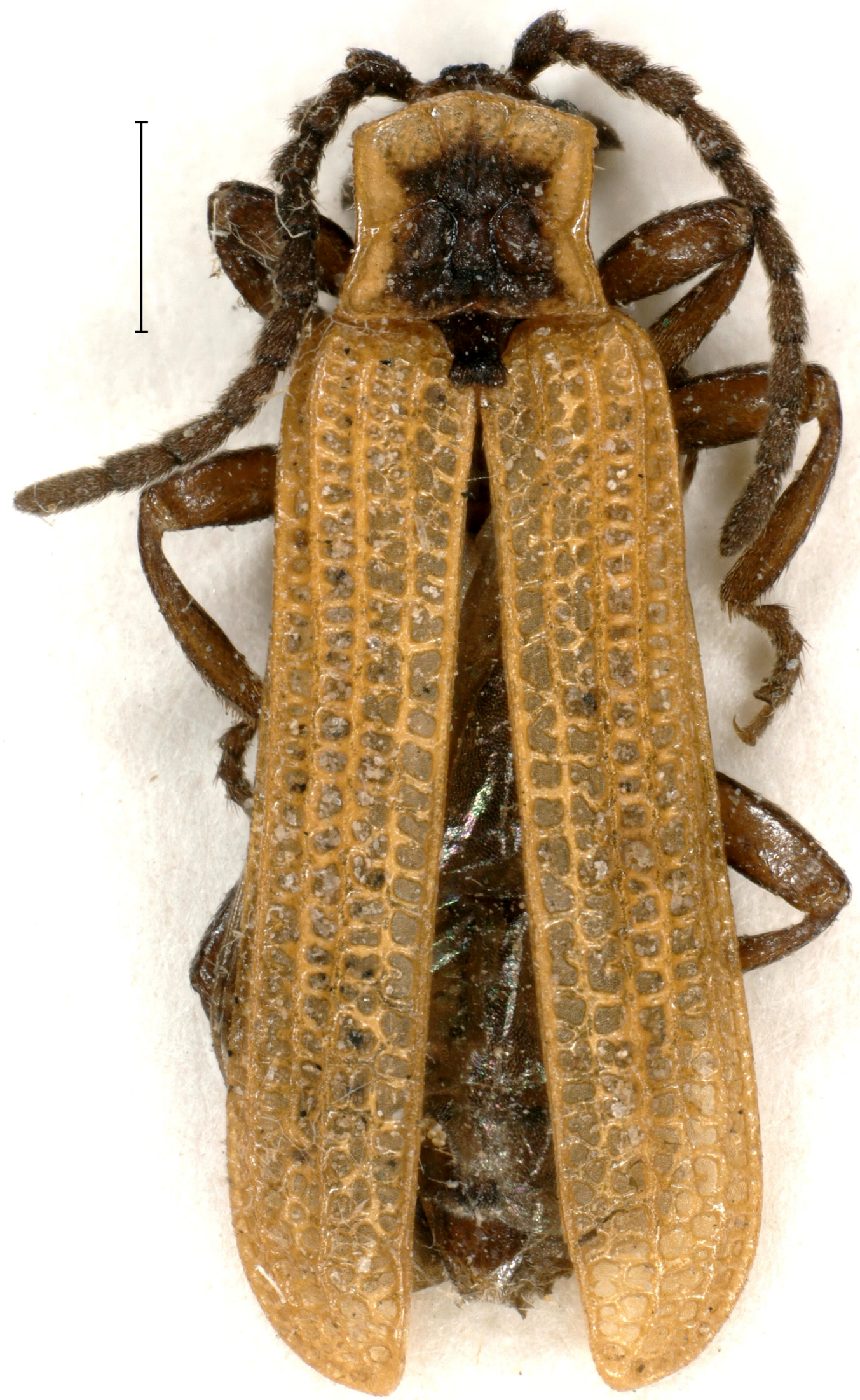

FIGURE 3. Erotides cosnardi (Chevrolat, 1831). Specimen preserved at the National Museum of Natural History of Luxembourg, scale bar $=1.0 \mathrm{~mm}$. 


\section{References}

Alexander, K.N.A. (2012) Cantharidae. In: Duff, A.G. (Ed.), Checklist of Beetles of the British Isles. $2^{\text {nd }}$ Edition. Pemberley Books, Iver, pp. 1-171.

Bocak, L. \& Bocakova, M. (1987) Notes on the taxonomy of some European species of the family Lycidae (Coleoptera). Acta Entomologica Bohemoslovaca, 84, 111-121.

Bocakova, M. \& Bocak, L. (2007) Catalogue: Family Lycidae Laporte, 1838. In: Löbl, I. \& Smetana, A. (Eds.), Catalogue of Palaearctic Coleoptera. Vol. 4. Elateroidea, Derodontoidea, Bostrichoidea, Lymexyloidea, Cleroidea, Cucujoidea. Apollo Books, Stenstrup, pp. 211-224.

Braunert, C. (1996) Faunistik, Ökologie und Gefährdung der Sandlaufkäfer (Coleoptera, Cicindelidae) Luxemburgs. Bulletin de la Société des naturalistes luxembourgeois, 97, 181-191.

Braunert, C. (2009) Verzeichnis der Rüsselkäfer Luxemburgs (Coleoptera, Curculionoidea) mit Ausnahme der Borkenkäfer (Scolytinae) und Kernkäfer (Platypodinae). Bulletin de la Société des naturalistes luxembourgeois, 110, $125-142$.

Braunert, C. \& Gerend, R. (1997) Checkliste der Laufkäfer (Coleoptera, Carabidae s. lat.) Luxemburgs. Bulletin de la Société des naturalistes luxembourgeois, 98, 169-184.

Bretzendorfer, F. (2002) Malthodes alpicola Kiesenwetter, 1852 (Col., Cantharidae) — Neu für Württemberg und Luxemburg. Mitteilungen des entomologischen Vereins Stuttgart, 37, 63.

Chevin, H., Ellis, W.N. \& Schneider, N. (2011) Mise à jour du répertoire des hyménoptères symphytes du Luxembourg (Insecta, Hymenoptera, Symphyta). Bulletin de la Société des naturalistes luxembourgeois, 112, 109-117.

Chittaro, Y., Sanchez, A. \& Geiser, M.F. (2021) An updated checklist of the Cantharidae and Lycidae of Switzerland (Coleoptera, Elateroidea). Alpine Entomology, 5, 77-94. https://doi.org/10.3897/alpento.5.67808

Constantin, R. (2014) Drilidae, Omalisidae, Lycidae, Lampyridae, Cantharidae (pp. 437-446). In: Tronquet, M. \& al. 2014 : Catalogue des Coléoptères de France. Association Roussillonnaise d'Entomologie éd., Perpignan, 1052 pp.

Diéguez Fernández, J.M. (2011) Nuevas citas y catálogo de los Cantharidae y Dasytidae (Coleoptera) del área iberobalear. Heteropterus Revista de Entomología, 11 (1), 75-85.

Diéguez Fernández, J.M. (2012) Nuevas especies y registros de Cantharidae del área iberobalear (Coleoptera). Heteropterus Revista de Entomología, 12 (1), 1-7.

Diéguez Fernández, J.M. (2014) Registros interesantes de coleópteros para España (Insecta: Coleoptera). 3a nota. Arquivos Entomolóxicos, 10, 119-124.

Everts, E. (1922) Coleoptera Neerlandica: de schildvleugelige insecten van Nederland en het aangrenzend gebied. Vol. 3. Martinus Nijhoff, 's-Gravenhage, xviii + [2] + 668 pp.

Fanti, F. (2014) Catalogo critico delle Cantharidae d'Italia (Insecta, Coleoptera). Memorie della Società Entomologica Italiana, $91(1-2), 61-132$. https://doi.org/10.4081/MemorieSEI.2014.61

Fanti, F. \& Vitali, F. (2013) Lygistopterus anorachilus Ragusa, 1883 (Coleoptera, Lycidae), morphological and faunistic remarks. Atti della Società italiana di Scienze naturali e del Museo civico di Storia naturale in Milano, 154 (1), 71-75. https://doi.org/10.4081/nhs.2013.71

Ferrant, V. (1917) Ueber den Massenfang des Rebenstechers an unserer Mosel. „Fauna“ Verein Luxemburger Naturfreunde, 27, 235-248.

Geisthardt, M. (2012) Fauna Europaea: Coleoptera, Lampyridae. Fauna Europaea, version 2.6.2 http://www.faunaeur.org (accessed 8 June 2021) [1.3 version of 2007]

Geisthardt, M. \& Satö, M. (2007) Catalogue: Family Lampyridae Latreille, 1817. In: Löbl, I. \& Smetana, A. (Eds.), Catalogue of Palaearctic Coleoptera. Vol. 4. Elateroidea, Derodontoidea, Bostrichoidea, Lymexyloidea, Cleroidea, Cucujoidea. Apollo Books, Stenstrup, pp. 225-234.

Gerend, R. (2000) Die Käferfauna eines Kalkmagerrasens über Steinmergelkeuper im Luxemburger Gutland: „Schléidelbierg“ bei Junglinster (Insectea: Coleoptera). Bulletin de la Société des Naturalistes luxembourgeois, 100, 103-134.

Gerend, R. (2003) Vorläufiges Verzeichnis der Wasserkäfer Luxemburgs (Coleoptera: Hydradephaga, Hydrophiloidea part., Dryopoidea part., Microsporidae, Hydraenidae, Scirtidae). Bulletin de la Société des naturalistes luxembourgeois, 104, 67-78.

Gerend, R. (2006) Erster Nachtrag zum Verzeichnis der Wasserkäfer Luxemburgs (Insecta, Coleoptera: Hydraenidae, Hydrophilidae, Scirtidae). Bulletin de la Société des naturalistes luxembourgeois, 106, 117-122.

Gerend, R., Köhler, F. \& Braunert, C. (2007) Die Totholzkäfer (Coleoptera) des “Schnellert” bei Berdorf: ökologische Analyse der Xylobiontenfauna eines Altwaldes in der luxemburgischen Sandsteinlandschaft. Ferrantia, 50, 265-295.

Hellers, M. (2016) Die Kleinschmetterlinge Luxemburgs: die Familien Micropterigidae, Eriocraniidae, Opostegidae, Heliozelidae, Adelidae, Prodoxidae, Incurvariidae, Tischeriidae und Tineidae. Bulletin de la Société des naturalistes luxembourgeois, $118,111-129$.

Hellers, M. (2017) Die Kleinschmetterlinge Luxemburgs: die Familien Roeslerstammiidae, Douglasiidae, Bucculatricidae und Gracillariidae (Lepidoptera: Gracillarioidae). Bulletin de la Société des naturalistes luxembourgeois, 119, $129-144$.

Hellers, M. (2018) Die Kleinschmetterlinge Luxemburgs: Die Familie der Palpenmotten (Microlepidoptera, Gelechiidae). Bulletin de la Société des naturalistes luxembourgeois, 120, 139-155. 
Hellers, M. (2019) Die Kleinschmetterlinge Luxemburgs: die Familien Yponomeutidae, Ypsolophidae, Plutellidae, Glyphipterigidae, Argyresthiidae, Lyonetiidae, Prayidae, Scythropiidae und Ethmiidae (Insecta, Lepidoptera). Bulletin de la Société des naturalistes luxembourgeois, 121, 153-168.

Hellers, M. (2020) Die Kleinschmetterlinge Luxemburgs: die Familien Depressariidae, Elachistidae, Agonoxenidae, Chimabachidae, Oecophoridae, Stathmopodidae, Batrachedridae, Momphidae, Blastobasidae, Autostichidae, Amphisbatidae, Cosmopterigidae, Schreckensteiniidae, Epermeniidae und Choreutidae. Bulletin de la Société des naturalistes luxembourgeois, 122, 103-125.

Horion, A. (1953) Faunistik der mitteleuropäischen Käfer. Band III: Malacodermata, Sternoxia (Elateridae bis Throscidae). Entomologische Arbeiten aus dem Museum G. Frey (Sonderband), München, 340 pp.

Kazantsev, S.V. (2004) A checklist of Cantharidae (Coleoptera) of the ex-USSR. Russian Entomological Journal, 13 (1-2), 23-34.

Kazantsev, S.V. (2012) Fauna Europea: Cantharidae. In: Karsholt, O., Nieukerken, E.J. van \& de Jong, Y.S.D.M. (2012): Fauna Europea: Coleoptera. Fauna Europea. Version 2.5. And last update 29 August 2013. Version 2.6.2. Available from: http:// www.faunaeur.org (accessed 8 September)

Kazantsev, S.V. \& Brancucci, M. (2007) Catalogue: Family Cantharidae Imhoff, 1856 (1815). In: Löbl, I. \& Smetana, A. (Eds.), Catalogue of Palaearctic Coleoptera. Vol. 4. Elateroidea, Derodontoidea, Bostrichoidea, Lymexyloidea, Cleroidea, Cucujoidea. Apollo Books, Stenstrup, pp. 234-298.

Köhler, F. (2009) Die Totholzkäfer (Coleoptera) des Naturwaldreservates „Laangmuer” In: Murat, D., Zoologische und botanische Untersuchungen „Laangmuer” 2007-2008. Naturwaldreservate in Luxemburg. Vol. 5. Naturverwaltung, Luxemburg, pp. 48-115.

Köhler, F. (2011) Die Totholzkäfer (Coleoptera) des Naturwaldreservates „Enneschte Bësch” (2007-2009). In: Murat, D., Zoologische und botanische Untersuchungen „Enneschte Bësch” 2007-2010. Naturwaldreservate in Luxemburg. Vol. 8. Naturverwaltung, Luxemburg, pp. 78-136.

Köhler, F. (2013) Die Totholzkäfer (Coleoptera) des Naturwaldreservates „Grouf” (2008-2009). In: Murat, D. — Zoologische und botanische Untersuchungen „Grouf” 2007-2011. Naturwaldreservate in Luxemburg. Vol. 10. Naturverwaltung, Luxemburg, pp. 50-107.

Magis, N. (1956) Faune entomologique du Grand-Duché de Luxembourg. III. — Cantharoidea (Col.). Musée d'Histoire Naturelle Luxembourg, Archives, Nouvelle Série, 23, 135-137.

Magis, N. (1968) Sur les Malacodermes paléarctiques 36-40. Bulletin et Annales de la Société Royale d'Entomologie de Belgique, 104, 152-163.

Magis, N. (1970) Coleoptera Cantharidae (cartes 89 à 100). In: Cartographie des Invertébrés Européens. Atlas provisoire des Insectes de Belgique. Cartes 1 à 100. Faculté des Sciences Agronomiques de l'Etat, Zoologie générale et Faunistique, Gembloux. [unknown pagination range]

Magis, N. (1971) Coleoptera Cantharidae (cartes 279 à 300). In: Cartographie des Invertébrés Européens. Atlas provisoire des Insectes de Belgique. Cartes 201 à 300. Faculté des Sciences Agronomiques de l'Etat, Zoologie générale et Faunistique, Gembloux. [unknown pagination range]

Magis, N. (1972) Contribution à l'étude monographique du genre Cantharis Linné, 1758 (Coleoptera: Cantharidae) II. Caractères morphologiques et morphométriques des espèces apparentées à Cantharis fusca Linné. Bulletin et Annales de la Société Royale Belge d'Entomologie, 108, 186-223.

Magis, N. (1976) Coleoptera Cantharoidea non Cantharidae (cartes 889 à 898). In: Cartographie des Invertébrés Européens. Atlas provisoire des Insectes de Belgique. Cartes 801 à 1000. Faculté des Sciences Agronomiques de l'Etat, Zoologie générale et Faunistique, Gembloux. [unknown pagination range]

Magis, N. (1977) Catalogue des Coléoptères de Belgique. Fascicule VI. Catalogue raisonné des Cantharoidea. Première Partie. Homalisidae, Drilidae, Lampyridae et Lycidae. Société royale belge d'Entomologie, Bruxelles, 60 pp.

Magis, N. (2005) Les Coléoptères Cantharoidea de la collection Guy Lhost. Notes fauniques de Gembloux, 56, 41-44.

Majka, C.G. \& MacIvor, J.S. (2009) The European lesser glow worm, Phosphaenus hemipterus (Goeze), in North America (Coleoptera, Lampyridae). ZooKeys, 29, 35-47. https://doi.org/10.3897/zookeys.29.279

Meyer, M. (1977) Groupe de travail pour l'étude des invertébrés. Arbeitsgruppe für Wirbelloseforschung: Entomologischer Bericht für die Jahre 1974 und 1975. Bulletin de la Société des naturalistes luxembourgeois, 80, 59-78.

Meyer, M. \& Pelles, A. (1981) Atlas provisoire des insectes du Grand-Duché de Luxembourg. Lepidoptera. 1ère partie. Ferrantia, $1,1-147$.

Meyer, M. \& Pelles, A. (1989) Éléments pour une révision faunistique des Macrolépidoptères du Grand-Duché de Luxembourg. 1. Les Rhopalocères de la collection C. Wagner-Rollinger. Päiperlék, 11 (2), 63-80.

Mousset, A. (1969). Les Coléoptères du Grand-Duché de Luxembourg. Bulletin de la Société des naturalistes luxembourgeois, 70, 139-174.

Mousset, A. (1981a) Les Coléoptères des sablières de Remerschen-Wintrage. Bulletin de la Société des naturalistes luxembourgeois, 83-84, 53-70.

Mousset, A. (1981b) Nova Coleopterologica. Bulletin de la Société des naturalistes luxembourgeois, 83-84, 71-73.

Niedringhaus, R., Biedermann, R. \& Nickel, H. (2010) Verbreitungsatlas der Zikaden des Großherzogtums Luxemburg — Atlasband. Ferrantia, 61, 1-395. 
Olm, K. (1892) Ausflug vom 6. Juni 1892. „Fauna“ Verein Luxemburger Naturfreunde, 2 (3), 36-37.

Proess, R. (2004) Verbreitungsatlas der Heuschrecken des Großherzogtums Luxemburg. Ferrantia, 39, 1-178.

Proess, R. (2006) Verbreitungsatlas der Libellen des Großherzogtums Luxemburg. Ferrantia, 47, 1-164.

Reichling, L. (2001) Atlas des hétéroptères non-aquatiques du Luxembourg. Musée national d'histoire naturelle, Luxembourg, $134 \mathrm{pp}$.

Silkin, N. (2008) Gazi Üniversitesi Zooloji Müzesi’nde Bulunan Cantharidae (Coleoptera) Örneklerinin Değerlendirilmesi. Gazi Üniversitesi Fen Bilimleri Enstitüsü, Ankara, 2008, 148 pp.

Švihla, V. (2006) Resurrection of Cantharis (Cyrtomoptila) fibulata (Coleoptera: Cantharidae). Studies and reports of District Museum Prague-East, Taxonomical Series, 2 (1-2), 123-127.

Švihla, V. \& Dvořák, L. (2009) Faunistic records from the Czech Republic — 281. Klapalekiana, 45, 208.

Vitali, F. (2012) Les espèces de Meloe L., 1758 au Grand Duché de Luxembourg (Coleoptera Meloidae). L'Entomologiste, 68 (1), 49-52.

Vitali, F. (2018) Atlas of the Insects of the Grand-Duchy of Luxembourg: Coleoptera, Cerambycidae. Ferrantia, $79,1-208$.

Wegnez, P., Ignace, D. \& Morro, C. (2021) Atlas des fourmis luxembourgeoises (Hymenoptera, Formicidae). Ferrantia, 84, $1-84$.

Zagatti, P. \& Mériguet, B. (2014) Présence de Cantharis paradoxa Hicker, 1960 dans le Nord de la France (Coleoptera Cantharidae). L'Entomologiste, 70 (6), Notes de Terrain et Observations Diverses, 373-374. 